\title{
Mariage et parentalité pour les couples de même sexe
}

\author{
Ann Robinson
}

Taking as her example the response of the Law to demands by same-sex couples for marriage and parental rights, Ann Robinson shows that the ethics of the Law, far from being motivated by rationality, is actually tied to a symbolic order which is highly interested. References by "right-thinking people" to nature or to what Robinson calls a "dogmatic anthropology" are countered by research carried out primarily in the United States, Belgium and England which undermines the absolute character of the latter references and shows them to be social constructions specific to the West.

Le mariage, institution patriarcale par excellence ${ }^{1}$ ! Mais aussi, le mariage cellule de base de la société et porte d'entrée à la famille dite légitime²! Au Canada comme dans la plupart des pays occidentaux, le droit au mariage est reconnu comme un droit de la personne, une liberté fondamentale. ${ }^{3}$ Mais alors comment se fait-il que des juristes surtout civilistes, des parlementaires et des juges (Borrillo « Fantasmes » 162) continuent de clamer bien haut l'in-accessibilité au mariage pour les lesbiennes et les gais ? La légendaire « rationalité » du droit serait-elle mise en échec par les opinions partisanes des bien-pensants, ceux et celles qui « font le monde »? Les enjeux sont grands, car au-delà du mariage, la famille aussi est en cause, de même que toutes les lois se rapportant à la famille, telles les lois sur la filation.

\section{Le droit au service des bien-pensants}

Le vent de « rectitude politique » qui souffle sur le monde occidental a entraîné au Canada et au Québec, comme partout ailleurs, une attitude et un discours marqués par la tolérance à l'égard des homosexuels. ${ }^{4}$ Cependant, lorsque l'on y regarde de plus près, cette tolérance se limite, la plupart du temps, à reconnaître l'orientation sexuelle des lesbiennes et des homosexuels. Non seulement on reconnaît leur marginalité, mais on voudrait les cantonner dans cette marginalité. ${ }^{5}$ Et lorsque les lesbiennes et les gais réclament le «droit à l'indifférence » dans le cadre de la 
reconnaissance de la conjugalité homosexuelle, l'intolérance reprend son droit de cité $^{6}$ même dans les rangs du groupe le plus libéral des scientifiques et des juristes.

S'il y a trente ans, les homosexuels se mobilisaient pour réclamer le droit de « sortir du placard », de vivre au grand jour, dorénavant, du fait de cette reconnaissance légale de non-discrimination, « socialement, l'ouverture du mariage et de la filiation aux couples de même sexe n'est donc plus impensable; elle est devenue seulement discutable (Fassin 92). On l'aura vite compris, pour un certain nombre de scientifiques, ce n'est pas le mariage des lesbiennes et des gais qui est en soi un problème, mais plutôt l'accès à la filiation qu'entraîneraient nécessairement ces mariages.

Même s'il y a encore quelques parlementaires ou juristes qui justifient le non-accès au mariage pour les homosexuels par les préceptes de la doctrine chrétienne, ${ }^{7}$ lesquels définissent l'homosexualité comme une dépravation et appellent à la chasteté les personnes atteintes de cette « tare » (Catéchisme de l'Église catholique 480) en invoquant parfois la nécessité de la «sacralisation de la filiation » (Fassin 95) dans une société laïque, la plupart de ces bien-pensants tentent plutôt de justifier leurs préjugés hétérosexistes par le biais de l'anthropologie, de la psychanalyse, de la sociologie ou même de la critique féministe de l'institution du mariage.

Pour un grand nombre de scientifiques que l'on ne peut qualifier a priori de conservateurs, l'hétérosexualité dans le mariage, c'est-à-dire la différence des sexes « aurait ainsi un fondement, non plus suprapolitique, mais infrapolitique - non plus théologique, mais anthropologique » (Fassin 96). On verse alors dans $l^{\prime}$ « anthropologie dogmatique », cette anthropologie qui traite non pas « des réalités sociales empiriques », mais plutôt des « dogmes qui instituent la société ». ${ }^{8} \mathrm{C}^{\prime}$ est alors que surgit le concept de $\mathrm{l}^{\prime}$ " ordre symbolique » anthropologique qui ne peut être maintenu que dans « la différence des sexes ». Ainsi, « [1]a loi de la différence des sexes, souveraine, doit toujours échapper à l'empire des lois » (Fassin 97). À l'instar des membres de la classe politique et des dirigeants de l'Église catholique qui répugnent à dénoncer ouvertement l'homosexua-lité au nom de la "rectitude politique " (Fassin 100), psychanalystes ${ }^{9}$ et sociologues ${ }^{10}$ ont emboîté le pas à ces anthropologues dogmatiques qui " prêchent » la différence des sexes ${ }^{11}$ comme l' ordre Symbolique » (Prokhoris 151) désormais immuable.

« Le mariage est le tombeau de la femme, le principe de toute servitude féminine ». Telle est la définition que donnait Charles Fourier du mariage 
au XIXe siècle (Borrillo Homosexualité 6). Mais, si cette définition pouvait faire image auprès de nos grand-mères et même de nos mères, elles qui ont vécu quotidiennement ce lieu d'oppression, il faut quand même admettre que ce n'est pas tant le mariage qui est « à l'origine de l'oppression des femmes, mais que c'est la société de type patriarcale [sic] et misogyne qui en est la cause » (Borrillo Homosexualité 6). Et d'ailleurs, dès le début du XXe siècle, partout dans le monde occidental, les législatures ont bien été forcées, poussées par les divers mouvements de femmes, de concéder graduellement l'égalité juridique aux femmes mariées. Ainsi au Québec, depuis l'adoption du fameux Bill 89 en 1980, « les époux ont en mariage, les mêmes droits et les mêmes obligations ${ } .^{12}$

Si un jour le mariage était légalisé entre deux femmes, pourrions-nous prétendre que cette institution serait encore un lieu d'oppression pour les femmes ? "Ou, au contraire, n'assisterions-nous peut-être pas pour la première fois à une véritable égalité dans le mariage " (Borrillo Homosexualité 6) ? En d'autres mots, lorsque les femmes ont commencé à s'émanciper et lorsque le législateur a dû suivre ce mouvement jusque dans les rapports privés entre époux, le mariage est graduellement devenu " une union ... entre deux êtres socialement égaux, qui se sont choisis librement et mutuellement pour satisfaire leurs besoins sentimentaux respectifs, et imposant d'égales obligations de fidélité aux deux partenaires » (Boswell 71).

Mais il ne faut surtout pas confondre le mariage avec le droit au mariage. En effet, toute personne, qu'elle soit homosexuelle ou hétérosexuelle, homme ou femme, scientifique ou juriste, conservatrice ou libérale, peut être contre le mariage. Mais de quel droit cette même personne pourra-telle s'opposer à l'accès au mariage pour une autre personne? Pour certains hétérosexuels, au nom de la « loi naturelle» (Pousson-Petit 406-07) ou de la « loi divine ${ }^{13}$ ? Ou pour certains homosexuels, au nom de la « différence » (Eribon 63-64) ? Au contraire, dans une société libre et démocratique qui préconise le multiculturalisme et le respect des différences, comme la société canadienne, il ne devrait pas être possible à qui que ce soit d'empêcher ne serait-ce qu'un seul couple homosexuel d'avoir accès au mariage, si tel est son désir.

\section{L'homoparentalité et l'accès au mariage pour les gais et lesbiennes}

La branche libérale des bien-pensants pourrait, semble-t-il, concéder le mariage aux gais et lesbiennes, si ce n'est que cette concession entraînerait 
sans doute le droit à l'homoparentalité. ${ }^{14}$ Bien sûr, dans les cas où des enfants sont pratiquement abandonnés par un ou leur deux parents hétérosexuels et que les seuls adultes dans leur entourage sont des lesbiennes ou des homosexuels, les tribunaux accepteront peut-être de céder à ces dernières et ces derniers la garde de ces enfants, malgré leur sexualité «marginale ». ${ }^{15}$ L'homosexualité n'est pas en soi un empêchement au droit de garde d'un parent. Ce slogan maintes fois répété dans la jurisprudence ${ }^{16}$ et dans la doctrine québécoise (D.-Castelli 174) ne porte en soi aucune avancée quant à l'homoparentalité. En effet, dans une recherche portant sur la jurisprudence québécoise quant à la question de la garde d'enfant associée au lesbianisme de la mère, ${ }^{17}$ il ressort que c'est seulement lorsque le juge ne peut pas faire autrement qu'il concède la garde de l'enfant à la mère lesbienne, par exemple, lorsque le père est incestueux ${ }^{18}$ ou lorsque ce que la mère offre comme confort et sécurité est largement supérieur à ce que peut offrir le père. ${ }^{19}$ Dans ce dernier cas, le juge a pris tout de même en considération le fait que la mère vivait avec sa compagne une relation asexuée et que l'enfant réclamait très sérieusement ce changement de garde (Robinson « Lesbiennes » 47).

Pourtant, des recherches faites principalement aux États-Unis, en Belgique et en Angleterre (Delaisi de Parseval 232) démontrent clairement que des enfants élevés par des parents homosexuels ne développent aucune pathologie particulière. Par exemple, aux États-Unis des études empiriques ont démontré qu'il n'y avait aucune différence significative quant au développement de l'identité sexuelle, au développement psychosocial ou à l'adaptation psychologique entre les enfants ayant des parents homosexuels et ceux ayant des parents hétérosexuels. ${ }^{20}$

Par ailleurs, la question de l'homoparentalité s'est complexifiée dans les dernières années sans doute parce que la société hétérosexuelle a développé et légitimé de nouvelles techniques de procréation médicalement assistée. Les couples de même sexe réclament maintenant le droit à la filia-tion par l'entremise de l'insémination artificielle et, par voie de conséquence, le droit à l'adoption. Mais, " [1]a filiation homoparentale suscite une véritable horreur » (Iacub 189) étant surtout « ressentie comme une menace pour la survie de la civilisation et de l'espèce humaine $"{ }^{21}$ En effet, juristes et scientifiques invoquent le caractère dit naturel des lois de filiation et prétendent « qu'il y aurait certaines structures anthropologiques immuables qui limiteraient la puissance normative des sociétés, telles que la différence des sexes et des générations " (Iacub 190). Mais si l'on y 
regarde de plus près, on constatera rapidement que les règles de filiation ne sont rien de plus qu'une construction sociale propre au monde occidental. ${ }^{22}$

En matière d'adoption le Code civil du Québec ne pose, de façon explicite, que deux principes : une " adoption ne peut avoir lieu que dans l'intérêt de l'enfant », 23 et « toute personne peut seule ou conjointement ... adopter un enfant $» .{ }^{24}$ Mais, il semble bien que dans la pratique quotidienne des organismes québécois d'adoption, « l'intérêt de l'enfant » va de pair avec l'hétérosexualité des parents adoptifs. Il a pu arriver à l'occasion qu'une personne homosexuelle, s'appuyant sur le principe qu'une personne seule peut adopter un enfant, ait pu en taisant son homosexualité, se voir confier un enfant pour adoption. Mais son conjoint ou sa conjointe ne pourra jamais prétendre à aucun droit de filiation à l'égard de cet enfant. Pourtant, en Ontario (Robinson « Lesbiennes » 54), et récemment en Alberta ( Des lesbiennes obtiennent » A-7), des tribunaux ont déjà prononcé des jugements d'adoption en faveur des conjointes des mères lesbiennes. Et quant à l'insémination artificielle qui n'est pas à proprement parler une technique de procréation médicalement assistée comme le laisse entendre le Code civil, ${ }^{25}$ certains juristes et bioéthiciens voudraient en restreindre l'usage, l'encadrer et surtout la réserver aux couples hétérosexuels stables en prétendant que c'est une " intervention thérapeutique visant à pallier l'infertilité des hommes membres de ces couples $» .{ }^{26}$

Mais au-delà de ces restrictions concernant l'adoption et l'insémination artificielle dans notre société québécoise, ce qu'il importe de considérer dans le cas actuel, c'est la situation du conjoint homosexuel ou de la conjointe lesbienne du père ou de la mère d'un enfant, que cet enfant soit né d'une relation hétérosexuelle antérieure, qu'il ait été adopté par une personne dite célibataire, ou qu'il soit né par l'entremise d'une procréation médicalement assistée. Dans chacun de ces cas, pourquoi affirme-t-on avec autant de force et de vigueur qu'il est dans l'intérêt de cet enfant, de n'avoir qu'un seul parent ? Est-ce au nom de cette nécessaire " différence de sexe » dans la parenté juridique, qui seule, crée, selon certains scientifiques, ${ }^{27}$ les règles de filiation? Mais alors, comment ces spécialistes expliquent-ils que des générations d'enfants depuis la nuit des temps et dans toutes les sociétés ont, dans les faits, été soignés, éduqués, élevés, gardés, par des femmes seules (Robinson « Lesbiennes » 49-50) ? Parce qu'elles étaient prétendument hétérosexuelles ? Le droit doit-il être à tout prix une fiction sans aucun lien avec les faits ${ }^{28}$ ? 


\section{Notes}

1 «Ainsi données, domestiquées, violées, louées, exploitées, exécutées au besoin, soumises à l'arbitraire de l'époux, entièrement subordonnées aux intérêts de celui-ci et radicalement privées de destin propre, telles apparaissent les épouses dans la législation napoléonienne. Si ce fleuron d'un patriarcat triomphant a dû progressivement laisser la place à des dispositions moins cruelles pour les femmes et moins dégradantes pour les deux sexes, l'esprit qui l'inspira est toujours vivace et fécond, il s'incarne aujourd'hui dans d'autres formes » (Dhavernas 42).

2 « Si le mariage est l'œuvre des clans et des lignages autant que des époux, il intéresse directement l'État en tant qu'institution fondamentale du système social $\gg$ (Dhavernas 28-29).

${ }^{3}$ Ann Robinson, Le mariage pour les gais et lesbiennes: Futur inéluctable. Rapport de recherche, Partie 2: «Le mariage dans l'histoire des pays de droit civil et de common law ».

${ }^{4}$ « La majorité des Canadiens ont foi dans la justesse du principe décrit dans la Charte des droits et consistant à veiller à ce que tous les Canadiens bénéficient d'un accès égal aux avantages et ne fassent pas l'objet de discrimination en raison de leur orientation sexuelle. Au cours des 20 dernières années, les Canadiens ont de plus en plus accepté le fait que l'homosexualité remonte à la naissance et ne constitue pas vraiment un autre mode de vie. C'est quelque chose que la nature ou Dieu nous a donné. C'est une imperfection ou peut-être même une anomalie. Il n'y a rien de contre nature dans une anomalie, car chacun d'entre nous est venu au monde avec des différences, des points faibles ou des points forts » (Bryden 16 010).

${ }^{5}$ Voir "L'essuie-misère ", entretien avec Pierre Legendre, dans Le Monde de l'éducation (1997): 37; rapporté dans Borrillo, Fassin et Iacub, Au-delà du PaCS : L'expertise familiale à l'épreuve de l'homosexualité (168).

6 « Si nous voulons empêcher les pédophiles, la drogue, la violence, les désordres à l'école et dans les banlieues, ne comptons pas seulement sur la police, les juges, les prisons et la castration chimique, ne détruisons pas les sentinelles invisibles qui maintiennent la cohérence de notre société : la différence entre l'homme et la femme et la stabilité de la famille. Ces projets portent atteinte à la cohésion sociale et à nos structures familiales.... Ils décomposent l'ensemble de notre droit civil, social et fiscal de la famille. Il ne faut les accepter en aucune manière » (Malaurie 879).

7 À preuve le député libéral Paul Szabo, qui au moment du débat sur la 
définition du mariage, à la Chambre des communes en juin 1999, invoque dans son discours une lettre de l'archevêque Ambrozic de Toronto. Voir Bryden, Hansard (15 983).

${ }^{8}$ Comme dans $l^{\prime}$ " anthropologie dogmatique ", étudiée entre autres par l'historien du droit Pierre Legendre, L'inestimable objet de la transmission, étude sur les principes généalogiques en Occident.

9 Pour Pierre Legendre par exemple, la sphère publique (l'État) doit «garantir les repères de la raison, c'est-à-dire assurer l'égalité de l'un et l'autre sexe dans la reproduction, sauvegarder les images de l'homme et de la femme, et en faire des images fondatrices du père et de la mère par le relais du droit.... Investir (dans le PaCS), c'est investir dans la violence de demain, la violence qui est toujours le lot de la perte des repères $\gg$ (ZuckerRouvillois 126). Voir également Tony Anatrella, La différence interdite.

10 «Ainsi, face aux revendications de parentalité homosexuelle, nos experts prétendent qu'il y aurait certaines structures anthropologiques immuables qui limiteraient la puissance normative des sociétés, telles que la différence des sexes et des générations " (Iacub 190).

11 « Dans le journal La Croix, interviewée en novembre 1998 par Marianne Gomez à propos du PaCS, l'anthropologue [Françoise Héritier-Augé] déclare : «Ce que j'ai essayé de montrer, c'est que la différence anatomique, physiologique et fonctionnelle des sexes - par fonctionnelle, j'entends le fait que c'est la femme qui porte les enfants - est à la base de la création de l'opposition fondamentale qui nous permet de penser. Car penser, c'est d'abord classer, classer c'est d'abord discriminer et la discrimination fondamentale est basée sur la différence des sexes. C'est un fait irréductible: on ne peut pas décréter que ces différences-là n'existent pas, ce sont des butoirs indépassables de la pensée, comme l'opposition du jour et de la nuit » (Zucher-Rouvillois 125).

${ }^{12}$ Article 392 C.c.Q.

13 «[Le] mariage est une communauté permanente entre l'homme et la femme, ordonnée à la procréation des enfants par quelque coopération sexuelle. Le mariage est une communauté qui a vocation à engendrer, ce ne peut être le désir d'une union homosexuelle » (Beignier 4).

14 « L'enjeu clairement énoncé de la loi sur le PaCS portait uniquement, on le sait, sur la question du couple, c'est-à-dire de l'alliance, et non de la filiation, soigneusement écartée du projet de loi. Or, la reconnaissance d'une filiation pour les couples homoparentaux - que ce soit par adoption ou par procréation médicalement assistée - a pourtant constitué le principal 
épouvantail, agité depuis les premiers débats parlementaires, avec le relais médiatique. Les fantasmes que suscite la question ont pu trouver un fondement dans l'argument de la " pente glissante », selon lequel «le couple homosexuel, une fois reconnu, exigera aussitôt d'être reconnu comme couple parental à part entière » (Delaisi de Parseval 225).

15 «Cependant, si le couple homosexuel est de plus en plus reconnu dans la société contemporaine, et il faut s'en féliciter, on peut se demander s'il peut équivaloir au couple mixte en tant que couple parental. Nous parlons ici bien sûr du cas où deux personnes du même sexe, recourant à la procréation médicalement assistée, revendiqueraient l'établissement légal de la filiation d'un enfant, qui serait ainsi reconnu comme fils ou fille de deux hommes ou de deux femmes. Nous ne parlons pas des cas particuliers qui peuvent conduire, dans les faits, deux hommes ou deux femmes, ou plus, quelle que soit la nature de leur relation, à élever ensemble un enfant. Ces cas sont toujours possibles et souvent heureux si les circonstances ont privé un enfant de ses parents. Cela ne pose pas de problème de principe si l'origine ou la filiation de l'enfant ne sont pas en cause. La question est celle du droit, donc des normes et des institutions, non des situations de fait » (Agacinski 117-18).

${ }^{16}$ Voir entre autres : Monette c. Sylvestre [1981] C.S. 731; Droit de la famille14 J.E. 83-135.

${ }^{17}$ Voir Ann Robinson, «Lesbiennes, conjointes et mères : Les exclues du droit civil québécois».

18 Droit de la famille - 316 J.E. 86-1127. Résumé de certains éléments de preuve, rapporté dans : Robinson, « Lesbiennes » 46.

${ }^{19}$ Droit de la famille - 1287 (1989) R.D.F. 625.

20 Voir Danielle Julien, Monique Dubé et Isabelle Gagnon, « Le développement des enfants de parents homosexuels comparé à celui des enfants de parents hétérosexuels »; Danielle Julien et Élise Chartrand, « La psychologie familiale des gais et des lesbiennes : perspective de la tradition scientifique nord-américaine ».

${ }^{21}$ Iacub, « Homoparentalité et ordre procréatif » 189, citant Irène Théry, « Le contrat d'union sociale en question », Esprit 10 (1997): 159-87.

22 «Et cependant, il n'est pas indispensable de se rapporter au tissu normatif des sociétés exotiques pour invalider ces postulats. Il ne faut même pas évoquer l'histoire si extravagante de nos propres droits occidentaux. Il suffit de comprendre le fonctionnement de l'ordre juridique pour refuser d'admettre l'existence des structures universelles qui 
prédétermineraient le contenu du droit » (Iacub 190-91).

${ }^{23}$ Article 543 C.c.Q.

${ }^{24}$ Article 546 C.c.Q.

${ }^{25}$ Article 538 C.c.Q.

${ }^{26}$ Commission des droits de la personne du Québec, De l'illégalité à l'égalité 116. Voir également : Iacub 195.

27 « La possibilité même de ces questions tient au fait que la notion de sexualité semble s'être complètement déplacée; elle concernait habituellement la différence des sexes dans son rapport à la génération. À la sexualité biologique répondait une « sexualité » sociale traduite par des institutions comme les alliances et la filiation. On croyait pouvoir superposer la distinction masculin/féminin aux distinctions mari/femme ou père/mère. La différence des sexes - et donc aussi les effets d'identités qu'elle produisait - était essentiellement liée à cette différence des rôles dans la génération, qui fit l'objet de tant d'interprétations. Ce fondement de la différence semble essentiel à Françoise Héritier qui ne situe nullement la différence des sexes - et ses enjeux - au niveau du sexe anatomique mais bien à celui de la génération et donc de la fécondité des femmes » (Agacinski 109).

28 « Dans le cas de la parenté adoptive, par définition, l'enfant n'est pas issu de l'union de ses parents, il n'est pas de leur chair; pourtant ses pa-rents sont pleinement institués dans leur état de parents soit en tant que couple marié, soit en tant que célibataires. Je pourrais parfaitement reprendre pour le modèle d'adoption plénière français ce que Françoise-Romaine Ouellette déclare pour le modèle québécois : "Il reflète directement notre modèle culturel de reproduction inspiré du processus biologique de la procréation sexuée, que j'appellerai ici modèle généalogique parce qu'il donne sa trame à notre système symbolique de la parenté. Ce modèle dicte une logique idéale d'établissement des filiations selon laquelle chaque individu est issu de deux autres individus d'une génération ascendante et de sexe différent qui l'auraient en principe conjointement engendré, ses père et mère." L'anthropologue québécoise ajoute que l'effet principal de ce modèle généalogique n'est pas tant "une biologisation de la parenté que l'imposition d'un principe exclusif de la filiation : chaque individu n'est en position de fils (ou de fille) que par rapport à un seul homme et à une seule femme, i.e. chaque enfant n'a qu'un seul père et une seule mère" » (Cadoret 215). 


\section{Ouvrages cités}

Agacinski, Sylviane. Politique des sexes. Paris: Seuil, 1998.

Anatrella, Tony. La différence interdite. Paris: Flammarion, 1998.

Beignier, Bernard. «Une nouvelle proposition de la loi relative au CUS; copie à revoir », « Droit de la famille ». Jurisclasseur (avril 1997).

Borrillo, Daniel. «Fantasmes des juristes vs Ratio juris : la doxa des privatistes sur l'union entre personnes de même sexe ». Au-delà du PaCS : L'expertise familiale à l'épreuve de l'homosexualité. Dir. Daniel Borrillo, Éric Fassin et Marcela Iacub. Paris: PUF, 1999.

---. Homosexualité et liberté matrimoniale. Version électronique. (18 nov 1999): 6.

Borrillo, Daniel, Éric Fassin et Marcela Iacub, dir. Au-delà du PaCS : L'expertise familiale à l'épreuve de l'homosexualité. Paris: PUF, 1999.

Boswell, John. Les unions du même sexe dans l'Europe antique et médiévale. Paris: Fayard, 1996.

Bryden, John, député libéral de Wentworth-Burlington. Débats de la Chambre des communes, (Hansard). Compte rendu officiel. Vol. 135, no 240. $1^{\text {re }}$ session, $36^{\mathrm{e}}$ législature.

Cadoret, Anne. «La filiation des anthropologues face à l'homoparentalité ». Borrillo et al.

Catéchisme de l'Église catholique. Mame/Plon. Paris: Édition du Vatican, 1982.

D.-Castelli, Mireille. « Garde d'enfants et relations extra-conjugales des parents ». 9 R.D.U.S. 165.

Delaisi de Parseval, Geneviève. « La construction de la parentalité dans les couples de même sexe» Borrillo et al.

Dhavernas, Odile. Droits des femmes, pouvoir des hommes. Paris: Seuil, 1978.

Eribon, Didier. Réflexions sur la question gay. Paris: Fayard, 1999.

Fassin, Éric. «La voix de l'expertise et les silences de la science dans le débat démocratique ». Borrillo et al.

Iacub, Marcela. « Homoparentalité et ordre procréatif ». Borrillo et al.

Julien, Danielle, et Élise Chartrand. " La psychologie familiale des gais et des lesbiennes : perspective de la tradition scientifique nord-américaine ». Sociologie et sociétés XXIX.1 (1997): 71-81.

Julien, Danielle, Monique Dubé et Isabelle Gagnon. " Le développement des enfants de parents homosexuels comparé à celui des enfants de parents hétérosexuels ». Revue québécoise de psychologie 15.3 (1994): $135-53$. 


$$
56 \cdot \text { Tessera }
$$

Legendre, Pierre. L'inestimable objet de la transmission, étude sur les principes généalogiques en Occident. Paris: Fayard, 1985.

«Des lesbiennes obtiennent le droit d'adopter l'enfant de leur conjointe ». Le Soleil (28 novembre 1999): A-7.

Malaurie, Philippe. «Un statut légal du concubinage? " Répertoire $d u$ notariat Defrénois 13-14 (juillet 1998) 879.

Pousson-Petit, Jacqueline. Le démariage en droit comparé. Bruxelles: F. Larcier, 1981.

Prokhoris, Sabine. «L'adoration des majuscules». Borrillo et al.

Robinson, Ann. Le mariage pour les gais et lesbiennes: Futur inéluctable. Rapport de recherche, Partie 2 : «Le mariage dans l'histoire des pays dew droit civil et de common law».

--. " Lesbiennes, conjointes et mères : Les exclues du droit civil québécois ». Des droits à reconnaître, les lesbiennes face à la discrimination. Montréal: Éditions du remue-ménage, 1998. 21-68.

Zucker-Rouvillois, Élisabeth. « L'expertise familiale ou la perte du doute scientifique ». Borrillo et al. 